such intolerant toxic symptoms that further experimentation was unjustified, and the last patient died tiio and a half months after treatment. While we did not feel that death resulted from the use of crotalin, the patient's disease certainly was not benefited by the treatment.

2005 Chestnut Street.

\section{THE NORMAL AND PATHOLOGIC PHYSIOLOGY OF THE VIS- CERAL NERVOUS SYSTEM}

WITH ESPECIAL REFERENCE TO VAGOTONY AND SYMPATIIICOTONY : A REVIEW *

JULIAN MAST WOLFSOHN, M.S., M.D.

Instructor in Neurology, Leland Stanford Junior Lniversity, Department of Medicine

SAN FRINCISCO

If one contemplates the contemporaneous literature, one is greatly impressed with the volume of work which is being done on the sympathetic nervous system.

It was Langley who, in the latter part of the nineteenth century, first called our attention to a more correct notion of the autonomic nervous system. He showed that the conception then current-that the sympathetic nervous system served wholly as a nerve pathway through which the socalled sympathetic actions of distant organs were affected and that it rid not, as Bell taught, arise from the brain by branches connected with the fifth and sixth cranial nerves-was wrong. His views in the main are those of the present-day conception.

ANATOMY AND PHYSIOLOGY OF TIIE VISCERAL NERVOLS SYSTEM

The autonomic nervous system, better termed the vegetative nervous system, is that system of efferent fibers arising from the sympathetic and related ganglia which supplies the organs of involuntary processes of the body and possesses a certain independence of the central nervous system. Its anatomy is too well known to be given here in detail. Briefly, it includes some of the cranial and sacral nerves and the sympathetic system proper. The latter is composed of a chain of ganglia lying on each side of the vertebral column. One ganglion, as a rule, is present to each spinal nerve root. In the cervical region only two ganglia are found, namely the superior and the inferior cervical ganglia. These are united by the cervical sympathetic trunk. The upper three to four thoracic ganglia are condensed to form the stellate ganglion. At the bottom of the chain only one coccygeal ganglion is found for the coccygeal vertebrae. All these constitute the first system or the "lateral ganglia."

'The second system of ganglia in the abdomen, called the "collateral ganglia," has a special connection

- Read before the San Francisco County Medical Society, Feb. 10 , 1914. with the abdominal viscera and includes the semilunar or solar ganglion, and the superior and inferior mesenteric ganglia.

The third system of ganglion cells, called the "terminal ganglia," including Auerbach's, Meissner's and the cardiac plexuses, is found in the viscera themselves. These have, as a rule, no connection with the fibers of the sympathetic system, but lie on the course of impulses descending by other nerves of the regetative system, for example, the vagus or pelvic visceral nerves. This system is capable of some inrlependence of the other nervous systems.

The sympathetic ganglia are connected with the spinal nerves just after they have given off their posterior divisions by means of rami communicantes, which are of two kinds: (1) white rami, consisting of small medullated fibers, and (2) gray rami, almost exclusively non-medullated fibers. The white rami are formed by fibers which have their origin in the spinal cord and perhaps in the posterior root ganglion. The gray rami represent fibers which, arising in the sympathetic ganglia, run back to join the spinal nerves.

The outflow represented by the white rami is limited from the first thoracic to the third or fourth

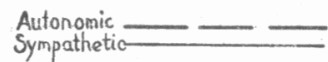
Vertebral Oculomotor nerve
Sanglion

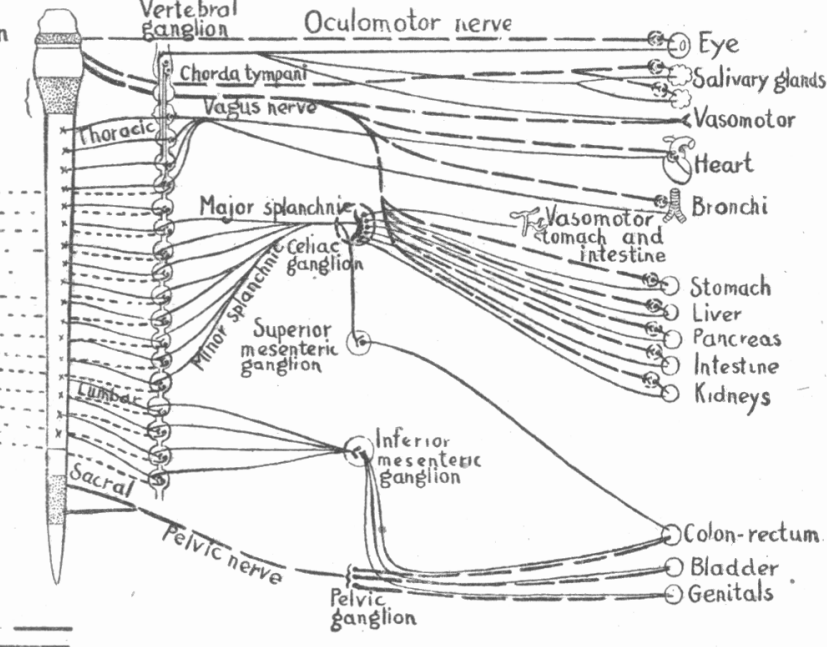


(myoneural, adenoneural) is present, in which the ions of the various elements exert their specific action.

Besides the main sympathetic nerves derived from the spinal cord region, certain cranial and spinal nerves, namely, the third, fifth, seventh, ninth and tenth cranial nerves and the nervi erigentes from the sacral region of the spinal cord, have visceral fibers. belonging to the vegetative nervous system, not directly connected with the sympathetic or the central nervous system. Those visceral fibers connected with the cranial nerves are termed the cranial autonomic nervous system, those in the sacral region, the sacral autonomic nervous system, the whole constituting the autonomic nervous system, sometimes termed the vagal system.

The visceral fibers of the third cranial nerve are called the midbrain autonomics. These pass as preganglionic fibers to the ciliary ganglion in the orbit, where they end. The postganglionic fibers from this ganglion form the short ciliary nerves which innervate the sphincter pupillae and ciliary muscles.

The visceral fibers connected with the other cranial nerves constitute the hindbrain autonomics.

The visceral fibers of the seventh cranial nerve arise from the medulla and intermediate nerve of Wrisberg (which is practically the anterior continuation of the ninth, tenth and eleventh cranial nerves), and give rise to the chorda tympani, which supplies the vasodilator nerves to the tongue, submaxillary and sublingual glands besides supplying secretory fibers to these glands. The preganglionic fibers to the sublingual and submaxillary glands end in the submaxillary ganglion.

The seventh cranial nerve also sends preganglionic visceral fibers to the sphenopalatine ganglion, whence the postganglionic fibers run with branches of the fifth cranial nerve to supply secretory and possibly vasodilator fibers to the mucous membrane of the nose, soft palate and upper pharynx.

The visceral fibers of the ninth cranial nerve, namely, the otic ganglion, produce vasodilatation and secretion of the parotid gland. Also a few dilator fibers go to vessels at the back of the tongue.

The visceral fibers of the tenth and eleventh cranial nerves arise in the same column of cells as the seventh and ninth. Most of these fibers run in the vagus, and include motor fibers to the esophagus, stomach and small intestines as far as the ileocolic sphincter and inhibitory fibers to the heart and smooth muscle of the bronchial and gastric glands. The preganglionic fibers end in the jugular ganglia. (It has been shown that the ganglia of the trunk of the vagus is probably the cell station for afferent fibers in this nerve.)

The nervus erigens contains all the pelvic visceral fibers and is connected with the ganglia lying in the hypogastric plexus. It has the following functions:

1. Dilator to the vessels of the penis.

2. Motor to the bladder, colon and rectum.

3. Inhibitory to the sphincter of the bladder.

4. Inhibitory to the retractor penis.

The afferent fibers of the visceral nerves probably arise from the cells of the posterior spinal ganglia.

In general it may be said, according to Langley, that the supply of afferent fibers of the viscera is very small in proportion to that of the outer surface of the body. For example, in the hypogastric nerve and also in the splanchnics, only about one-tenth of the medullated fibers are afferent. In the mouth, ants, lung and bladder, where both somatic and visceral innervation are represented, the afferent visceral supply becomes much greater in proportion; for instance, the pelvic visceral nerve contains about onethird afferent fibers.

Notwithstanding their afferent nerve-supply, ordinary stimulation of the viscera, such as handling, etc., produces no sensation of pain to the patient; but pathologic stimulation, such as stretching, compression and other effects of disease, is productive of pain which, as Head and Mackenzie have shown, is not referred to the diseased viscus, but is referred to certain parts of the cutaneous surface, hypersensitiveness of which localizes in large measure the position of the diseased viscus.

In describing the visceral fibers coursing with certain cranial nerves, the functions of these fibers was hinted at. The fibers of the vegetative nervous system, it may be stated generally, are not equivalent in their functions; for example, the heart-beat is quickened by stimulation of the accelerators (sympathetic) and slowed by stimulation of the vagi (autonomic). Again, the functions of the vagus for the greater part

\section{TABLE 1.-EFFECTS OF ELECTRIC STIMULATION}

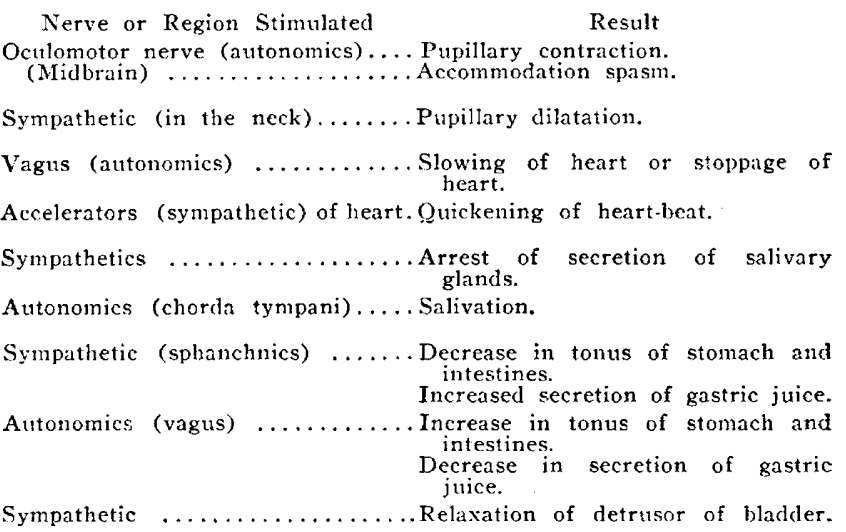

Autonomics (pelvic visceral nerve). Contraction of detrusor of bladder.

of the gastro-intestinal canal are motor, while those of the splanchnics (sympathetic) are inhibitory. These two examples not only show that the functions of the two sets of visceral fibers are antagonistic to each other, but they also cite what is true for most of the viscera: that generally the viscera are innervated by both the craniosacral (autonomic) nervous system and also by the sympathetic nervous system with functions reciprocal to each other. The functions, moreover, of any one set of fibers for a given viscus are not always sharply outlined; for instance, the colonic branches of the superior mesenteric ganglion are motor (constrictor) for the blood-vessels and inhibitory for the muscular walls of the colon; also the sympathetic, being inhibitory to the intestines generally, is also motor to the ileocolic sphincter, and sends both motor and inhibitory fibers to the bladder. Again, the nervi erigentes are not only dilator to the vessels of the penis and motor to the colon, bladder and rectum, but inhibitory to the sphincter of the bladder and to the retractor penis.

The adequate stimuli capable of producing effects through the visceral nervous system may be summed up under the following captions: 
1. Electric stimuli.

2. Mechanical stimuli.

3. Chemical stimuli, including those of :

A. Exogenous origin.

B. Endogenous origin: (a) hormones, $(b)$ internal secretions, (c) toxins, etc.

4. Emotions (probably ultimately chemical).

1. The effects of electric stimulation are given in Table 1.

2. Mechanical stimulation of the same nerves causes in general the same effects as produced by electric stimulation.

3. Chemical substances, whether exogenous or endogenous, are found to exert their influence on the vegetative nervous system, sometimes affecting one system exclusively, sometimes another, and sometimes both.

A. Exogenous chemicals:

1. Nicotin interrupts conduction at the junction (synapse) of the preganglionic and postgangionic fibers of both the autonomic and sympathetic systems.

2. Pilocarpin, muscarin and physostigmin and digitalis stimulate the vagus system (autonomics) and are thereforc called vagotropic or vagotonic drugs. The signs produced by these drugs are similar to those produced by electrical stimulation of the autonomics, namely, myosis, bradycardia, salivation, etc.

3. Atropin, scopolamin and other products of the belladonna series produce their effects by paralysis of the terminals of the vagus system (autonomics), especially the cranial autonomics, and are termed vagoparalytic drugs. The well-known effects of administration of these drugs-for instance, mydriasis, dry mouth, tachycardia, etc.-are very similar to the effects produced by stimulation of the sympathetic.

4. Picrotoxin has been shown to stimulate the preganglionic fibers of the autonomic system (vagus system).

5. Ergotoxin has a jaralytic effect on the terminals of some of the sympathetic fibers (sympatheticoparalytic), and can be compared to the effects of atropin, etc., on the autonomic terminals.

6. Cocain stimulates all the sympathetic fiber terminals, producing mydriasis and exophthalmos; the effects of cocain are not produced after degeneration of the sympathetic fibers. It has also been shown that cocain sensitizes the sympatnetic fibers to epinephrin (which see), and that so-called cocain poisoning is not really the effects of cocain, but rather an epinephrin effect.

B. Endogenous chemicals, hormones, toxins, etc.

7. Epinephrin stimulates the sympathetic system only at the terminal of the postganglionic fibers. It is sympathicotropic or better, sympathicotonic in action, and its effects are similar to those produced by electric stimulation of the sympathetic system.

8. Iodothyrin stimulates the sympathetic, especially in the cervical and thoracic regions.

9. Pituitrin causes vasoconstriction other than renal, and also produces contractions of the bladder and uterus.

10. Cholin (extracted from the cortex of suprarenal glands) produces results similar to pilocarpin, that is, vagotonic.

11. Recently Voegtlin and Macht isolated from the cortex of the suprarenal bodies a sympathicotonic substance whose effects were longer lasting than those of epinephrin.

12. The internal secretion of the pancreas favors inhibition of the sympathetic and is antagonistic to epinephrin, that is, vagotonic.

Many other less important substances could be included in this iist, but we can at once see that electric stimuli and certain chemical stimuli are iden- tical in their effects for a given set of fibers, and also that when organs are doubly innervated a certain effect can be produced by either the stimulation of one set (for instance, the sympathetic), or by paralysis of the other (that is, the vagus). Again it might be well to cite an example of this: a dilated pupil could be produced by paralysis of the vagus system or by stimulation of the sympathetic system in the neck (both of which conditions could, as stated above, be produced by chemicals).

Epitomizing the effects of chemical stimulation on the vegetative nervous system, we can make the classification given in Table 2 .

4. Cannon and de la Paz have shown that psychic excitement and fright are associated with stimulation of the vegetative nervous system and are capable of producing: (1) sympathicotonic effects such as pallor, mydriasis, tachycardia, and (2) vagotonic effects such as epiphora, sweating, etc.

Grouping all the foregoing effectual methods of stimulation of the vegetative nervous system together, it can readily be seen that the effects of various stimuli to the autonomic system (vagus system), and to the sympathetic system are in general specific and reciprocal.

SICNS OF VEGETATIVE NERVOUS SYSTEM DISORDERS

When one considers the wonderful balance which is maintained, not in the peripheral nervous system

TABLE 2.-EFFECTS OF CIIEMICAL STIMULATION

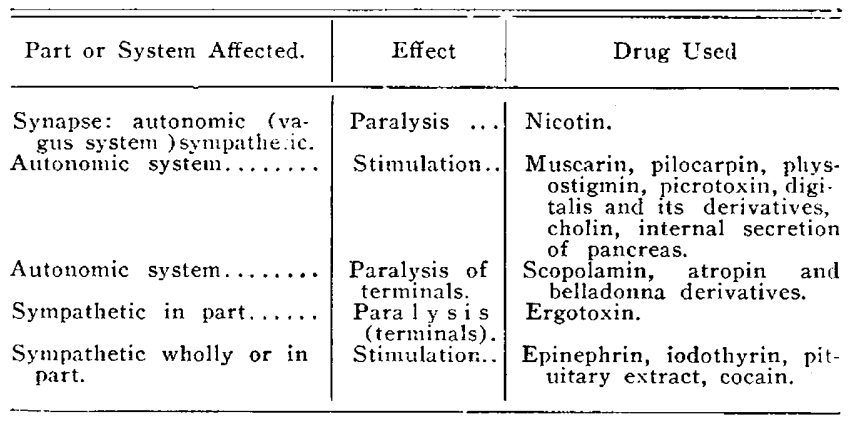

only (as evidenced by the action of opposing muscles in the acts of walking, standing, etc.), but especially in the vegetative nervous system, examples of which are manifold (as the equality of the pupils and the effects of light and other stimuli; the tone of the capillary system, and the rate of the heart-beat and its various rate modifiers), he is likely to give to the latter-named system the clinical weight which it so richly deserves, but which, for lack of definite knowledge of the effects on it of various agencies such as chemicals, ductless glands and metabolic disturbances on the various organs, is now being actively pursued.

The neurologist of to-day must needs fasten his attention, not alone on the study of the central nervous system, but also on the study of the vegetative nervous system-visceral neurology. How many are the cases which, when studied in this light, yield data which have helped to decide the correct and logical course of treatment instead of the formerly muchabused empirical course?

At this point it may be well to call attention to a rentmeration of some of the more important symptons and signs which the visceral neurologist must take heed of, given in Table 3 . 
Many other examples could be cited, but Table 3 constitutes a good framework on which one can build a classification of certain groups of diseases showing a predomination of vagotonic or sympathicotonic signs. Many of these signs and symptoms can be produced by decreased functioning of one of the systems with corresponding overfunctioning of the other; for example:

Tachycardia could be produced by paralysis of the vagus or overexcitation of the accelerators (sympathetic), etc.

Acrocyanosis, by paralysis or paresis of the vasoconstrictors to the skin.

Low blood-pressure by lessened sympathetic tonus:

Pigmentation of the skin in decreased sympathetic tonus.

\section{IMPORTANT SYMPTOMS AND SIGNS}

Vagotonic Symptoms and Signs

1. Myosis.

2. Accommodation spasm

3. Epiphora.

4. Hyperhidrosis

5. Frequency of winking.

6. Salivation with constant spit-

7. Hyperacidity of stomach con-

8. Arrest of secretion of gastric glands (achylia).

9. Hypermotility of stomach and intestines.

10. Vomiting. 12. Spastic colon.

14. Biliary colic (inhibited by atropin).

16. Enophthalmos

17. Bradycardia.

18. Low blood.pressure.

20. Pollakiuria.

22. Asthmatic attacks.

23. Esophagism.

24. Mucous colitis (ana

25. Gastrospasm and pylorospasm.

27. Dermatographia.

23. Outspoken status thymicoOutspoken status thy
lymphaticus.

30. Eosinophilia

31. Pulsus irregularis respiratorius, which disappears torius, which
with atropin.

2. Irregular breathing (disappears with atropin). pears with atroping.eet.

34. Priapisin.

35. Aschners' phenomenon produces slow pulse.

36. After atropin-pressure on eyeballs produces no slowing of pulse.

37. Increased fat tolerance.

38. Increased carbohydrate tolerance before and after epinephrin administration.

39. Pilocarpin causes extreme salivation.

Sympathicotonic Symptoms and Signs

1. Mydriasis.

2. Paralysis of accommodation.

3. Dryness of eyeballs.

4. Dryness of skin.

5. Infrequency of winking (Disl. rymple's sign).

6. Dryness of mouth.

7. Low gastric acidity.

8. Increased gastric secretion

9. Lessened intestinal tonus.

11. Constipation.

13. Faulty convergence of eyes (Moebius' sign).

\section{Wide eye-slits. \\ 16. Exophthalmos. \\ 18. High blood-pressure. asoconstriction as seen in (a) peripheral anemias, $(b)$ intermittent \\ 20. Relax etc. bladder (incontinence) \\ 21. Urticarita}

\section{Atony of stomach. \\ 26. (iastroptosis. \\ 27. No dermatographia \\ 28. Tonsils small and atrophic. \\ 29. Gag-reflex marked. \\ 30. Eosinopenia.}

\section{Tachypnea with dyspnea (not affected by atropin \\ 33. Dry hands and feet. \\ 35. Aschners' phenomenon pro- duces no change in pulse.}

37. Steatorrhea.

38. Lowered carbohydrate toler ance before and after epinephrin administration (i. e. epinephrin hypersensitive ness)

39. Pilocarpin causes no saliva-

40. Loewi test

\section{TESTS}

The following tests are used in the physiologic and pharmacodynamic studies:

1. Loewi's Test.-From 2 to 3 drops of a $1: 1,000$ epinephrin solution, instilled into the cye of a normal person, produces vasoconstriction but no pupillary dilatation. In some forms of hyperthyroidism, especially with lowering of autonomic sensibility, a pupillary dilatation occurs. This is also produced in pancreatic diabetes, that is, hypersusceptibility of pupils to epinephrin.
- 2. Aschner's Phenomenon-Pressure on the eyeballs for from one-half to one minute produces no change of pulse in normal persons. When vagotonia exists there is a rather sudden slowing of the pulse with this procedure. (This effect is caused by pressure on the eyeballs stimulating the trigeminus and reflexly the vagus, stimulation of which causes bradycardia and stoppage of respiration in the expiratory phase.)

3. Pilocarpin Test--Pilocarpin, 1/10 grain (hypodermically), causes extreme salivation and bronchial secretion in vagotonia. No salivation is noted in sympathicotonia.

4. Fat Tolcrance.-The normal fat tolerance is from 100 to $150 \mathrm{gm}$. in twenty-four hours. The patient is given 200 gm. of butter, with meals, to be eaten in twenty-four hours. The stools are saved and examined for fat for forty-eight hours following. Fat-free stools indicate a vagotonic condition. Marked steatorrhea indicates a sympathicotonia.

5. Carbohydrate Tolcrance.-Normally $100 \mathrm{gm}$. of glucose can be taken on a fasting stomach without a resulting glycosuria. In sympathicotonia a spontaneous glycosuria is produced, whereas an increased tolerance for glucose is present in vagotonia. The test is performed in the following manner:

On the fasting stomach $100 \mathrm{gm}$. of glucose with 400 c.c. of water are given at $6 \mathrm{a} . \mathrm{m}$. The patient immediately urinates and hourly thereafter for five hours. The specimens are to be saved separately and examined for glucose. The first specimen is used as a control. The tolerance for carbohydrates can be determined then by increasing or decreasing the amount of glucose at each test until a faint reduction to Fehling's solution is produced.

6. Epinephrin Hypersensitiveness.-One hundred gm. of glucose by mouth and $1 \mathrm{mg}$. of epinephrin hypodermically are given from a quarter to half an hour later, and the carbohydrate tolerance test, just described, followed out.

In vagotonia no glycosuria is produced by this procedure. In sympathicotonia about $6 \mathrm{gm}$. of glucose are excreted in the urine in five hours.

7. Pulsus Irregularis Respiratorius (Hering).-On deep inspiration and on expiration only slight normal physiologic difference is noted in the rate and size of the pulse. In vagotonic states, inspiration causes increase in the rate of the pulse and decrease in the size; expiration causes slower pulse and increase in size (best shown by tracings).

8. Dermatographism.-A. After blushing (Polonski) produced by rubbing the skin, pathognomic of loss of equilibrium in the vegetative nervous system, probably deficient sympathetic tonus.

B. "Perverse reaction" (Hess and Konigstein).

Rubbing the skin produces stimulation of the pilomotor fibers and vasoconstriction, that is, sympathicotonia. Patients in this group usually have pallor of the face which gets paler under psychic stimuli (whereas the normal result should be blushing).

To attempt a clinical classification at this time with the foregoing data is without the scope of this paper, but it would not be amiss to give in general the results of observation in twenty-three cases studied from the point of view of vagotonia and sympathicotonia. These cases include 11 of exophthalmic goiter, 6 of neurasthenia, 1 of bronchial asthma, 3 of hyperthyroidism, and 2 of gastric ulcer.

In general, it was found that one case reacted according to the vagotonic or to the sympathicotonic classification only. Just one case of gastric ulcer could be considered pharmacodynamically and symptomologically vagotonic alone.

'The hyperthyroidism and neurasthenic cases portrayed as many vagotonic as sympathicotonic signs, and were just as sensitive to epinephrin as to pilocarpin.

One case of bronchial asthma, clinically vagotonic, reacted violently to epinephrin with marked rigor, 


\section{THE SURGICAL TREATMENT OF POST- OPERATIVE PALATE DEFECTS}

This patient was also pilocarpin-sensitive. Atropin had no therapeutic effect on the attack.

Patients with exophthalmic goiter follow the clinical classification described in the outline, and a very interesting fact was noted in these cases. The region most affected, the eye, heart, etc., was more sensitive pharmacodynamically than were others not so implicated. When tachycardia was marked, epinephrin caused greater relative increase of the tachycardia than it did on the blood-pressure or tremor when these were not so marked. One case showed 2 per cent. cosinophilia, after pilocarpin; this increased to 5 per cent.

The chief symptoms produced in patients sensitive to pilocarpin were: marked salivation and sweating, lowered blood-pressure and slight nausea, and capillary dilatation, increase in surface temperature sometimes amounting to 1 degree. In one case the pulse was slowed. Eosinophilia was noted twice in the serics.

Epinephrin-sensitive patients reacted with tremor, tachycardia, increased blood-pressure (in one case by $20 \mathrm{~mm}$. Hg), glycosuria and rigors.

Hemmeter has emphasized that "patients who already have an organic disease of the stomach will answer more readily to chemicals that excite the secretions and motions of the stomach than normal individuals." The same can be said of the heart, kidney, skin, ductless glands and the general nervous system.

The subject of vagotonic and sympathicotonic states is still in its infancy, and the sharp lines drawn by the originators of this conception will in time be so nodified that a clearer conception of disease entities due to disturbances of the vegetative nervous system will be ferreted out.

Neuroses of various kinds, ductless gland disorders, vicious cycles, etc., will then be classified into rational clinical and pharmacologic bases. The fruits of these studies will not only enrich the fields of normal and pathologic physiology, but will also help to dispose of many fallacious conceptions existing in these realms.

Visceral neurology has come to stay. It will be found a stalwart weapon for attack on the diagnosis of many obscure conditions now classified under neurasthenia, neuroses, etc. The etiology of many common diseases, such as gastric ulcer, cardiospasm, constipation, ductless gland disturbances, etc., will be cleared up, thereby permitting a rational method of therapy.

At some future date an exposition of the cases will be given, and it is hoped that this paper will serve to stimulate active work in this fascinating subject.

I am greatly indelsted to Dr. R. L. Wilbur for the use of patients in his wards.

2502 Fillmore Street.

Heredity.-Heredity is not, as is often imagined by. bioiogists, a necessary property of living matter, comparable to the inertia of matter, a sort of biologic postulate which does not require a genetic explanation for itself. It is, like all biologic phenomena, a product of evolution; it is an adaptation, the prototype of all adaptations. Moreover, it is of fundamental importance, because without it life itself could not have been preserved, nor could there be any stability of form or of mechanism.--Lugaro, Problems in Psychiatry.
GEORGE V. I. BROWN, M.D.

Oral Surgeon St. Mary's Hospital and Children's Free Hospital

MILWAUKEE, WIS.

Acquired openings through the palate, if surgical or due to accidental injury, show an active tendency to repair which quite often restlts in the ultimate closure of fissures that under other conditions might not be expected to close without surgical operative interference. When these lesions occur as a result of syphilis, the hard-palate defects show remarkable ability to close themselves if the parts are kept properly clean and protected by a suitably adjusted plate; but when luetic ulcerations have destroyed any portion of the velum the characteristic contractions of scar tissue usually so deform the soft palate that it suffers not only the ill effects due to the tissue actually lost, but also still greater injury through the deformity, which of ten is exceedingly difficult to overcome when restoration is attempted.

Aside from considerations pertaining to the nature of the cause of the palate fissure, and those which concern the constitutional treatment necessary to preparation for operation in individual cases, the actual surgical requirements do not differ materially from those demanded in closure of the postoperative palatal defects of congenital cases.

Bearing in mind these exceptions we may therefore include them all in the following classifications:

1. Contraction and arrested development of the hard palate, associated with intranasal and extranasal defects and irregularities of the teeth due to forcible compression in an effort to close the palate fissure in early infancy.

2. Defects in the anterior portion of the hard palate augmented by imperfect labial connection in this region, as found almost invariably when the premaxilla has been removed or destroyed in closure of double harelip, and sometimes when the premaxillary portion of the palate has not been perfectly joined to the other maxillary divisions in infant operations.

3. Openings through the central portion of the hard palate alone.

4. Opening at the junction of hard and soft palates involving both.

5. Hole in the central portion of the velum palati.

6. Defect occurring on one side at the posterior border of soft palate.

8. Imperfect form of the faucial arches due to defective operative results.

9. Velum too short.

10. More than one defect, including any of the forms previously described.

Each of the foregoing conditions may be subject to many clinical subdivisions with reference to form and situation, and each requires distinct recognition in determining the operative steps to be followed in attempting their correction. Failure in this respect means almost invariably an ineffectual result. The measure of an operator's success in the treatment of these cases, presenting as they often do, histories of many previous unsuccessful attempts to close such palatal fissures completely, will be determined largely by his ability to detect and overcome the underlying opposing factors in each case, and the accuracy with
7. Fissure between the uvulae. 